\title{
Recurrent atypical meningioma seeding to surgical scar
}

\footnotetext{
Sir,

Meningiomas are common intracranial extra-axial tumors and are benign in character. Surgery is considered curative and the recurrence rate for Simpson's Grade 1 resection is less than $10 \%$. In $0.1 \%$ of the cases, meningiomas may show extra-cranial metastasis, occurring mostly in the lungs, liver, and lymph nodes. ${ }^{[1]}$ We report a rare case of atypical meningioma seeding to a surgical scar from a previous craniotomy.
} 
A 48-year-old lady presented with a 2-month history of left side body weakness and headaches. On examination, she had left hemiparesis and papilloedema. Magnetic resonance imaging (MRI) showed a right temporoparietal lesion having a thin dural tail and homogenous enhancement with gadolinium [Figure 1]. The patient underwent a right temporoparietal craniotomy and Simpson's Grade I excision of the lesion. Post-operative scans showed no residual tumor [Figure 2]. Histopathology revealed atypical meningioma (World Health Organization [WHO] Grade II). The patient returned to us after 1 year with recurrent left hemiparesis. Repeat MRI scans showed tumor recurrence. A re-exploration craniotomy was carried out and a Simpson Grade I resection was again completed. She made an uneventful recovery and histopathology again showed features of atypical meningioma (WHO Grade II).

A follow-up examination 6 months after the second surgery showed no local recurrence. However, there was a lump overlying the scar in an area unrelated to underlying burr holes or tumor resection margin

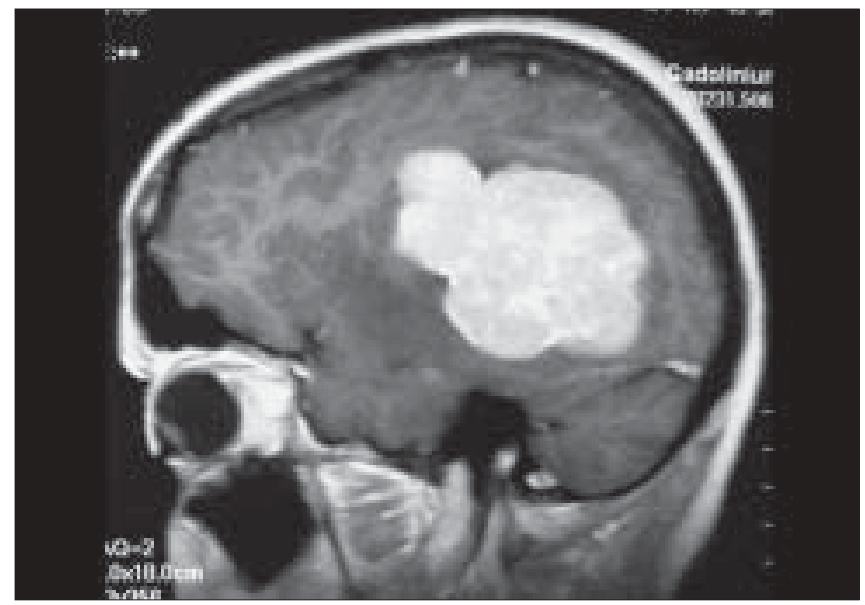

Figure 1: Pre-operative MRI scan (T1WI) saggital images with gadolinium enhancement showing left parietal convexity meningioma

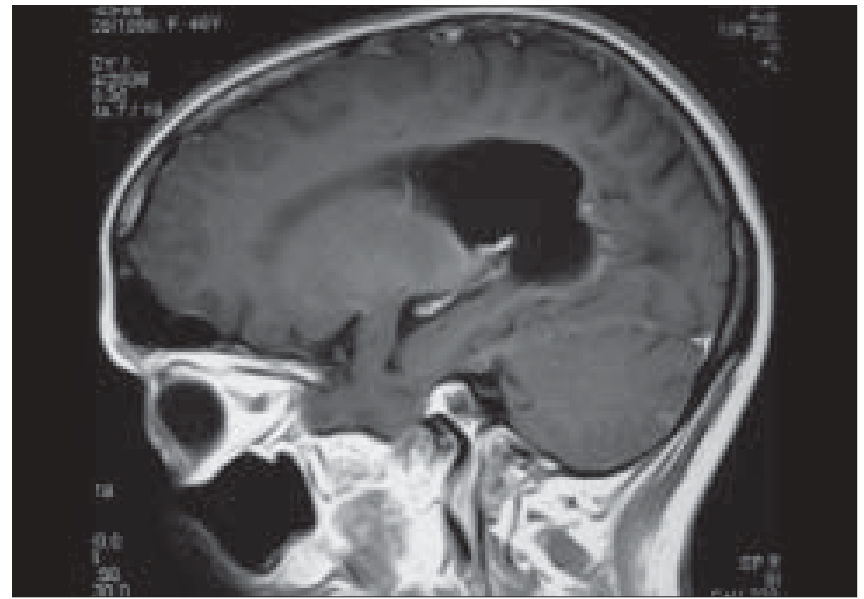

Figure 2: Post-operative MRI scan (T1WI) showing no residual tumor
[Figure 3]. A repeat computed tomography (CT) scan showed no evidence of intracranial recurrence but a small soft tissue extra cranial lump was obvious [Figure 4]. The lump was excised and sent for histopathology, which confirmed it to be metastasis from atypical meningioma [Figure 5]. Follow-up visits for 2 years were unremarkable.

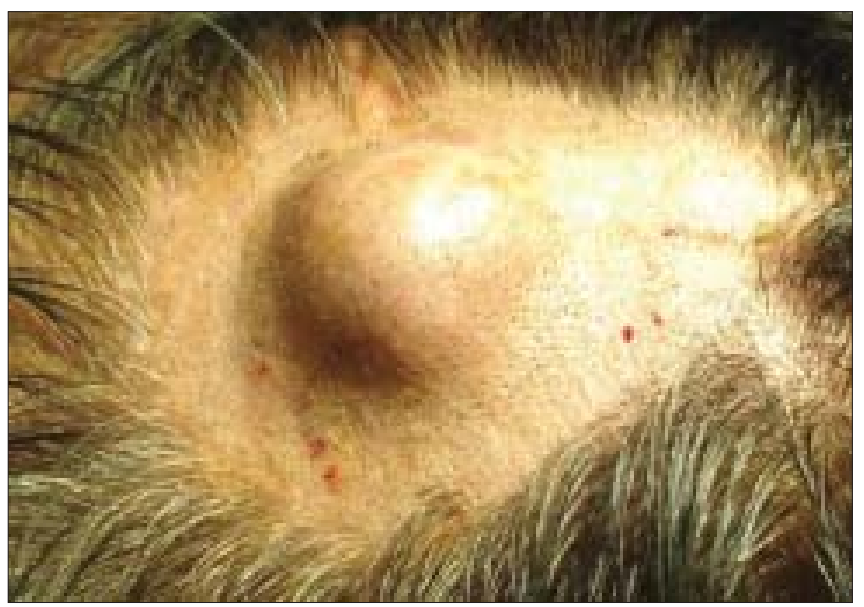

Figure 3: Visible subcutaneous lump

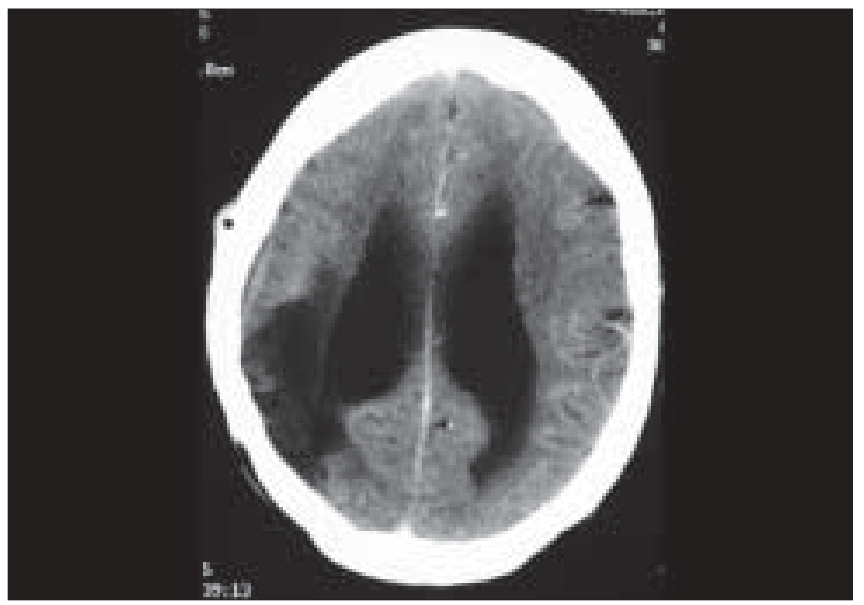

Figure 4: CT scan at third presentation showing no residual tumor, but a small soft tissue lump over the craniotomy site (marked with black dot)

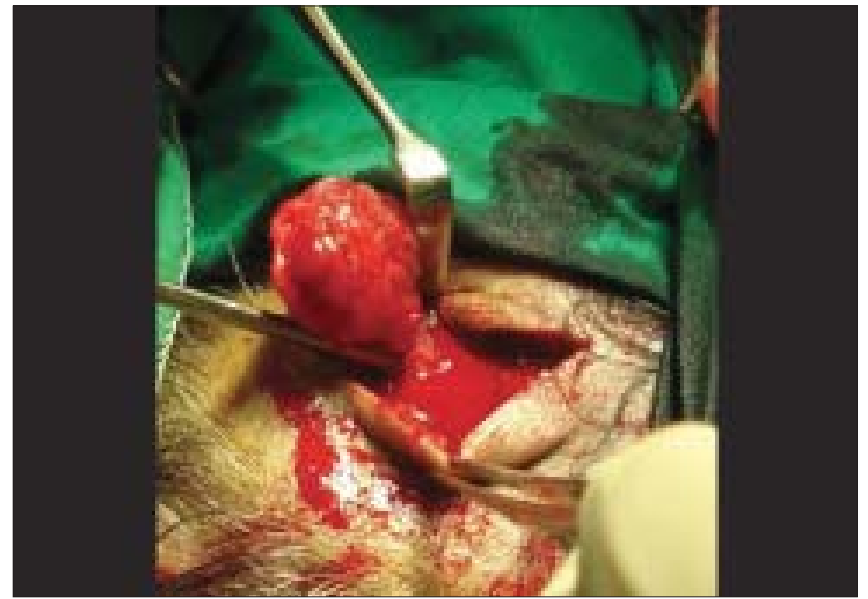

Figure 5: Intra-operative picture showing excision of lump 
Meningioma is a tumor that rarely metastasizes. Metastasis can occur through CSF, blood, or lymphatic spread. However, in our case, iatrogenic spread of the tumor cells during surgery resulted in skin seeding just under the scar of the previous incision and had no relation with the original lesion. To our knowledge, only 5 cases of iatrogenic seeding of meningioma have been reported. Of these, for 3 cases the site was the scalp and for the remaining two, it was the temporalis muscle and the graft donor site in the abdomen. ${ }^{[2-6]}$ Ludeman, et al., reported a case of meningioma in an 11-year-old boy of the right frontal region involving the frontal bone. ${ }^{[3]}$ Six months after surgery, subcutaneous metastasis developed over the scalp. Akai, et al., reported a case of a 70-yearold woman with meningioma of the falx extending to the frontal bone that recurred in ethmoid sinus 2 years after the resection and was removed through a transfacial approach. ${ }^{[2]}$ Six months later, the patient presented with scalp metastasis under skin incision. Ozer, et al., report a case of a 45-year-old female with peritorcular meningioma who underwent a resection. ${ }^{[6]}$ Two years later, she developed a subcutaneous lump over pin site, which on exploration, proved to be metastases. All 3 cases were of atypical meningioma on histopathology and received radiotherapy. Our patient did not receive radiotherapy at any stage of treatment. It is difficult to suggest possible reasons for such seeding but cell dissemination during surgery seems to be the most plausible explanation.

In conclusion, atypical meningiomas carry a risk of dissemination during surgery. Neurosurgeons should be aware of the possibility of iatrogenic seeding of the tumor, which may present as scalp swelling.

\section{Muhammad Z. Tahir, Muhammad S. Shamim, Khalid N. Chishti \\ Department of Neurosurgery, Aga Khan University Hospital, Karachi, Pakistan. E-mail: doctorneuron1@yahoo.com \\ DOI: $10.4103 / 0028-3886.51307$}

\section{References}

1. Karasick JL, Mullan SF. A survey of metastatic meningioma. J Neurosurg 1974;39:206-12.

2. Akai T, Shiraga S, Iizuka H, Kishibe S, Ueda Y. Recurrent meningioma with metastasis to the skin incision-Case Report. Neurol Med Chir (Tokyo) 2004;44:600-2.

3. Ludemann WO, Obler R, Tatagiba M, Samii M. Seeding of malignant meningioma along a surgical trajectory on the scalp. J Neurosurg 2002;97:683-6.

4. Singh RVP, Yeh JS, Campbell DA. Implantation meningioma in temporalis muscle-Case Report. Br J Neurosurg 1994;8:93-5.

5. Sadahira Y, Sugihara K, Manabe T. Iatrogenic implantation of malignant meningioma to the abdominal wall. Virchows Arch 2001;438:316-8.

6. Ozer E, Kalemci O, Acar UD, Canda S. Pin site metastasis of meningioma. Br J Neurosurg 2007;21:524-7. 